\title{
Solid or Liquid? Solidification of a Nanoconfined Liquid under Nonequilibrium Conditions
}

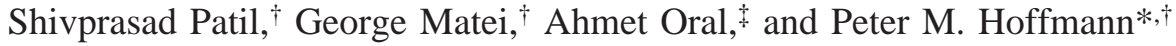 \\ Department of Physics and Astronomy, Wayne State University, 666 West Hancock, Detroit, Michigan \\ 48201, and Department of Physics, Bilkent University, 06800 Ankara, Turkey
}

\section{Received February 21, 2006. In Final Form: May 18, 2006}

\begin{abstract}
There has been a long-standing debate about the physical state and possible phase transformations of confined liquids. In this report, we show that a model-confined liquid can behave both as a Newtonian liquid with very little change in its dynamics and as a pseudosolid, depending solely on the rate of approach of the confining surfaces. Thus, the confined liquid does not exhibit any confinement-induced solidification in thermodynamic equilibrium. Instead, solidification is induced kinetically when the two confining surfaces are approached with a minimum critical rate.

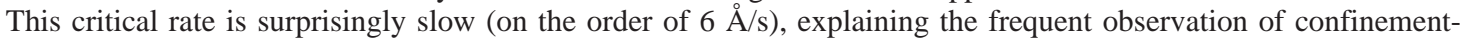
induced solidification.
\end{abstract}

\section{Introduction}

The structure and dynamics of confined liquids is of great importance in interfacial phenomena from cell membranes to nanotribology. ${ }^{1}$ In liquid films close to a flat solid surface, molecular layering is observed, ${ }^{2-4}$ which is enhanced by confinement between two solid surfaces. New tools to study confined liquids include surface force apparatus (SFA), ${ }^{2,5,6}$ atomic force microscopy (AFM), ${ }^{7,8}$ and spectroscopic techniques ${ }^{9}$ such as fluorescence correlation spectroscopy (FCS). ${ }^{10}$ Various experiments have yielded mutually exclusive findings on the dynamics of these confined systems. In octamethylcyclotetrasiloxane (OMCTS), a nonpolar, roughly spherical, "model" liquid, different research groups have reported behavior ranging from crystallization to glass formation to no transition at all. ${ }^{11-14}$ The same is true for water, the primary biological solvent. ${ }^{15-17}$ Here, we report on measurements of the nanomechanics and dynamics of confined OMCTS, performed with a novel AFM technique that allows for linear measurements of the stiffness and the damping coefficient of the confined liquid layer and is capable of separating the effects of cantilever frequency and approach rate.

* To whom correspondence should be addressed. E-mail: hoffmann@ wayne.edu.

'Wayne State University.

$\doteqdot$ Bilkent University.

(1) Bhushan, B.; Israelachvili, J. N.; Landman, U. Nature 1995, 374, 607.

(2) Israelachvili, J. N. Intermolecular and Surface Force; Academic Press: San Diego, CA, 1992; Chapter 13.

(3) Yu, C.-J.; Richter, G.; Datta, A.; Durbin, M. K.; Dutta, P. Phys. Rev. Lett. 1999, 82,2326

(4) Donnelly, S. E.; Birtcher, R. C.; Allen, C. W.; Morrison, I.; Furuya, K.; Song, M.; Mitsuishi, K.; Dahmen, U. Science 2002, 296, 507.

(5) Israelachvili, J. N.; McGiuggan, P. M. Science 1988, 241, 795.

(6) Granick, S. Science 1991, 253, 1374

(7) O'Shea, S. J.; Welland, M. E.; Pethica, J. B. Chem. Phys. Lett. 1994, 223, 336.

(8) Patil, S.; Matei, G.; Hong, H.; Hoffmann, P. M.; Karaköse, M.; Oral A. Rev. Sci. Instrum. 2005, 76, 103705.

(9) Heuberger, M.; Zäch, M.; Spencer, N. D. Science 2001, 292, 905.

(10) Mukhopadhyay, A.; Zhao, J.; Bae, S. C.; Granick, S. Phys. Rev. Lett. 2002, 89, 136103.

(11) Kumacheva, E.; Klein, J. Science 1995, 269, 816. Klein, J.; Kumacheva, E. J. Chem. Phys. 1998, 108, 6996.

(12) Demirel, A. L.; Granick, S. Phys. Rev. Lett. 1996, 77, 2261. Demirel, A. L.; Granick, S. J. Chem. Phys. 2001, 1115, 1498.

(13) Zhu, Y.; Granick, S. Langmuir 2003, 19, 8148.

(14) Mugele, F.; Becker, Th. Phys. Rev. Lett. 2003, 91, 166104.

(15) Zhu, Y.; Granick, S. Phys. Rev. Lett. 2001, 87, 096104.

(16) Raviv, U.; Laurat P.; Klein J. Nature 2001, 413, 51.

(17) Jeffery, S.; Hoffmann, P. M.; Pethica J. B.; Ramanujan, Ch.; Özer, H. Ö.; Oral, A. Phys. Rev B 2004, 70, 054114.

\section{Experimental Section}

The home-built AFM used in this study incorporates a fiber interferometer to measure changes in the cantilever's amplitude and phase. ${ }^{8}$ To perform a measurement, the AFM cantilever is vibrated far below the resonance frequency, and amplitude and phase are monitored using a lock-in amplifier as the sample is approached toward the tip. By using small cantilever amplitudes (smaller than the size of a single molecule), the measurements are linearized, allowing us to directly relate the measured cantilever phase and amplitude to the stiffness and the damping coefficient of the confined liquid. ${ }^{17}$ The sample consisted of OMCTS confined between a flat silicon oxide surface and a gold-coated ${ }^{18}$ silicon AFM tip. We also performed measurements between the same type of tip and other substrate surfaces, including mica and gold-coated mica. The tips were carefully cleaned using methanol and deionized (DI) water, but were otherwise not modified. The OMCTS was purified by passing it through molecular sieves and filtering it through a $20 \mathrm{~nm}$ pore size syringe filter just prior to each measurement. Any contamination of the OMCTS with moisture led to a strong attractive background in the force-distance curves, and such measurements were discarded. The silicon oxide surfaces were prepared by oxidation in a heated Piranha solution $\left(1: 3 \mathrm{H}_{2} \mathrm{O}_{2}, \mathrm{H}_{2} \mathrm{SO}_{4}\right)$ and subsequent drying in an oven at $120^{\circ} \mathrm{C}$ overnight. Experiments were performed at room temperature $\left(25^{\circ} \mathrm{C}\right)$.

\section{Results and Discussion}

Figure 1a shows the stiffness and the normalized damping coefficient of confined OMCTS measured at an approach rate of $3 \AA / s$ on a silicon oxide substrate. The damping coefficient is normalized to the measured bulk value far from the surface. This value varied between different levers, but was generally in the range of $10^{-5} \mathrm{Ns} / \mathrm{m}$. Clear stiffness and damping oscillations can be seen with an average separation of about $9 \AA$, consistent with the diameter of OMCTS molecules. The stiffness and the damping coefficient are "in-phase"; that is, the maxima of the stiffness are aligned with the maxima in the damping coefficient. Since a higher stiffness implies an increased density of the confined liquid, the confined liquid acts like a Newtonian fluid, where the viscosity is expected to increase with density.

To model the dynamic behavior of the liquid, we used the simplest viscoelastic model for a liquid: the Maxwell model. ${ }^{19}$

(18) Micromasch CSC37/Cr-Au cantilevers

(19) Findley, W. N.; Lai, J. S.; Onaran, K. Creep and Relaxation of Nonlinear Viscoelastic Materials; Dover: New York, 1976; Chapter 5. 

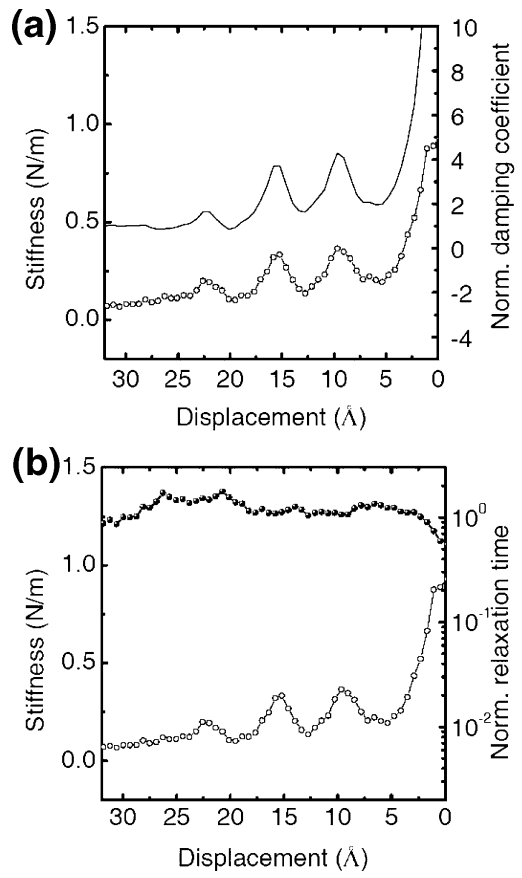

Figure 1. (a) Junction stiffness (open circles) and normalized damping coefficient (line graph) versus the displacement of OMCTS confined between the AFM cantilever tip and a silicon oxide surface. The cantilever frequency and free amplitude were $460 \mathrm{~Hz}$ and 3.5 $\AA$, respectively. The sample was approached toward the tip at a "slow" rate of $3 \AA / s$. The stiffness and the damping coefficient are in-phase in this case. (b) Junction stiffness (open circles) and Maxwell mechanical relaxation time (filled circles) versus displacement. Note that the relaxation time does not systematically change with displacement.

It consists of a linear spring and linear viscous element in series and exhibits time-dependent stress dissipation under application of an external strain. The characteristic relaxation time is given by $t_{\mathrm{R}}=k /\left(\gamma \omega^{2}\right)$, where $k$ is the measured junction stiffness, $\gamma$ is the measured damping coefficient, and $\omega$ is the oscillation (angular) frequency of the cantilever. In a liquid, stresses will dissipate quickly, and $t_{\mathrm{R}}$ is expected to be low. In ideal (elastic) solids, stresses can be sustained indefinitely, so $t_{\mathrm{R}}$ is expected to be large if the system behaves more like a solid.

Figure $1 \mathrm{~b}$ shows the normalized mechanical relaxation time of the liquid, $t_{\mathrm{R}}$, calculated from the data in Figure 1a. Again, the relaxation time is normalized to the measured bulk value, which typically was on the order of $2 \times 10^{-4} \mathrm{~s}$. The mechanical relaxation time does not show any systematic changes associated with either confinement or layering. Thus, the dynamics of the system seems to be unaffected by confinement, and the liquid remains liquidlike, even at small separations. The only change that is induced is a density oscillation as a function of separation associated with the geometrically induced layering of the liquid molecules.

In contrast, Figure 2a shows stiffness and damping at an approach rate of $12 \AA / s$. We can see a dramatic change in the relative magnitude of the stiffness and the damping coefficient. They are now "out-of-phase", and the liquid shows reduced damping in the "ordered", high stiffness state, and liquidlike damping in the disordered, low stiffness state. This suggests that, in the ordered state, the liquid now behaves more like an elastic solid. Figure $2 \mathrm{~b}$ shows the corresponding relaxation time, which now shows clear oscillations, with prominent maxima associated with the "solidlike", ordered state of the liquid.

Figure 3 summarizes our measurements. We plotted our observations in a matrix of the oscillation frequency of the
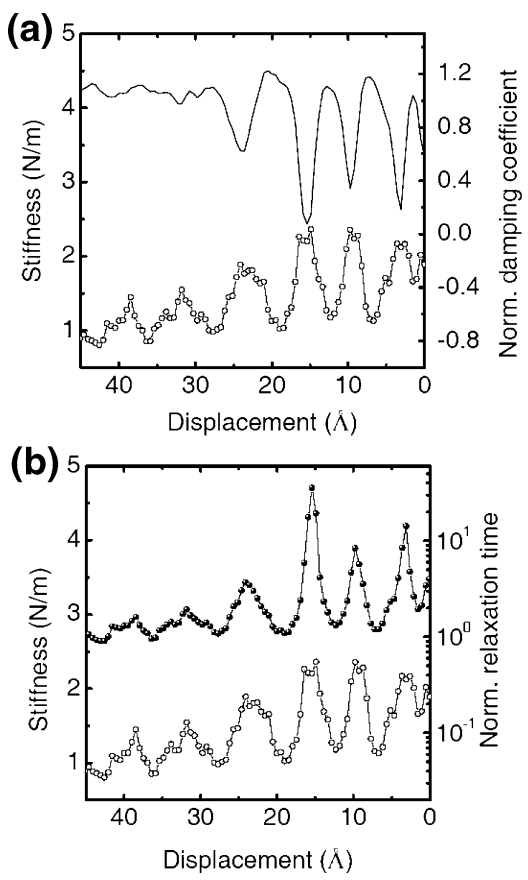

Figure 2. (a) Junction stiffness (open circles) and normalized damping coefficient (line graph) versus the displacement of OMCTS confined between the AFM cantilever tip and a silicon oxide surface. The cantilever frequency and free amplitude were $400 \mathrm{~Hz}$ and 2.4 $\AA$, respectively. In this case, the sample was approached toward the tip at a "fast" rate of $12 \AA / \mathrm{s}$. The stiffness and the damping coefficient are out-of-phase with respect to each other. (b) Junction stiffness (open circles) and Maxwell mechanical relaxation time (filled circles) versus displacement. Note that the relaxation time shows strong peaks associated with the high stiffness regions of the sample.

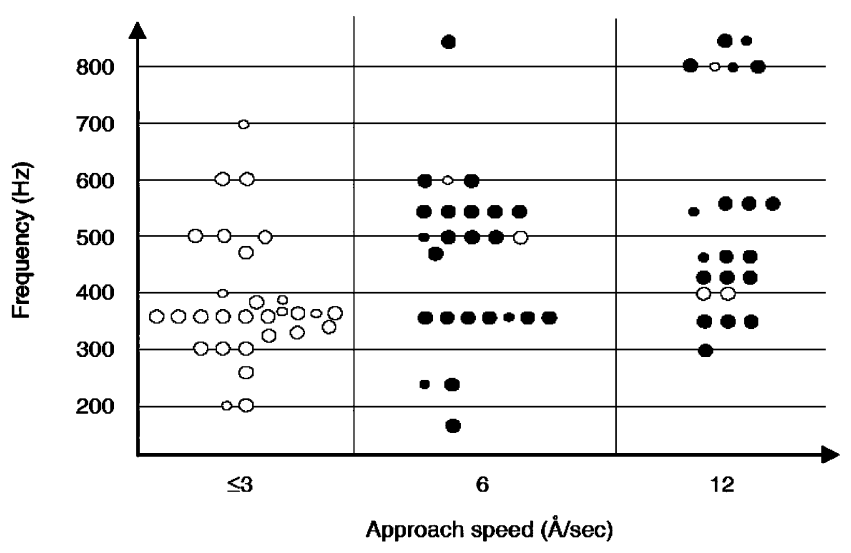

Figure 3. Summary of all measurements that showed stiffness oscillations plotted in a matrix of approach speed and cantilever oscillation frequency. It can be seen that liquid- or solidlike behavior depends merely on the approach speed and not on the oscillation frequency of the cantilever.

cantilever and the approach rate. In our experiments, we also explored different cantilever amplitudes in a restricted range from 1.5 to $7 \AA$, but, except for an attenuation of the peak heights in the stiffness at larger amplitudes, we did not find any systematic dependence in this range of small amplitudes. The open circles in Figure 3 denote cases in which stiffness and damping were in-phase, and the mechanical relaxation time was essentially constant with displacement, as in Figure 1 (liquidlike behavior). The filled circles denote measurements where stiffness and damping were out-of-phase, and the mechanical relaxation time showed distinct peaks associated with the high stiffness phase of the confined fluid, as in Figure 2 (solidlike behavior). The size 
of circles denotes the confidence in data: small circles denote cases where the stiffness and the damping peaks were aligned either in-phase or out-of-phase, but the data was noisy or the alignment of the peaks changed as surface was approached (in these cases the alignment closer to the surface was used). In all other cases (large circles), the relative alignment of the stiffness and the damping peaks was clearly either in-phase or out-ofphase over the full range of observed peaks. It should be noted that, because of the local roughness of the sample, not all measurements showed stiffness oscillations. The typical "success" rate in our measurements was on the order of $20 \%$.

As can be seen in Figure 3, the approach rate, rather than the oscillation frequency of the lever, is the crucial parameter that determines the dynamical behavior of the liquid. This may seem surprising since the maximum speed of the cantilever during each oscillation cycle is on the order of $1000 \AA / \mathrm{s}$, that is, much larger than the approach speed. However, the lever is oscillated at small amplitudes, compressing the liquid film only slightly without squeeze-out of a complete molecular layer. Thus, the oscillation of the lever probes the mechanical and dynamical properties of the confined liquid film, and the slow approach of the tip actually forces molecular layers to be pushed out of the tip-sample gap. We can thus postulate that the observed kinetic phase transformation may be due to the fact that, at a rate of 6 $\AA / \mathrm{s}$ and above, the molecular layers are "jammed" and are not able to react to the narrowing of the gap fast enough.

It is useful to compare our results with the recent experiments by Xhu and Granick, ${ }^{13}$ who report the onset of strong friction in confined OMCTS layers only if the layers were squeezed at a rate exceeding $5 \AA$ s. If the surfaces were approached much slower, friction was immeasurably small. Thus, the confined liquid became "solidified" at approach rates greater than or equal to $5 \AA / s$, almost identical to our observations. If we define a critical time scale by dividing the thickness of one molecular layer by the critical approach rate, we find a critical time on the order of $9 \AA /(6 \AA / s)=1.5 \mathrm{~s}$, which is $12-14$ orders of magnitude longer than typical molecular relaxation times. The identical time-scale observed in the SFA experiments by Xhu and Granick ${ }^{13}$ and our present observations, despite the vastly different lateral dimensions of the confined region $(\sim 10 \mu \mathrm{m}$ versus $\sim 10 \mathrm{~nm})$, suggest that this behavior is independent of the lateral size of the confined region. Rather, it must be intrinsic to the characteristic properties of the confined liquid and the thickness of the film (number of layers).

At this point, it is important to ask, How universal is this behavior? Is it unique to OMCTS, or do other liquids show similar behavior? As of now, because these are the first reports of such behavior in a simple liquid, we do not know the answer to this question. Another question is how much this behavior depends on the nature of the confining surfaces. Here, we performed some measurements on other substrates, namely, hydrogen-terminated silicon, mica, and gold films on mica. We found that the roughness of the film has a strong influence on the observed ordering. Even a small amount of random roughness of more than $3 \AA$ root mean square (RMS) over a $500 \mathrm{~nm}$ square can destroy measurable ordering (for this reason, we could not observe ordering on hydrogen-terminated silicon). However, our measurements on silicon oxide, mica, and gold-coated mica suggest that the chemical nature of the surface does not influence the observations described in this paper. This is also corroborated by the fact that Xhu and Granick found almost identical behavior, despite the fact that they used two clean mica surfaces, while we performed most of our measurements between a gold-coated silicon tip and an oxidized silicon wafer.
There are few models describing the squeeze-out dynamics of confined liquids. Persson et al. ${ }^{20}$ proposed a nucleation model. However, we found that the critical parameters obtained from this theory (particularly the pressure) do not match well with our experimental observations. Moreover, these parameters do not change much if the approach rate is varied in the range of 3-12 $\AA / s$ because of the logarithmic dependence of the critical values on the nucleation rate. Thus, this approach cannot explain the dramatic shift in behavior when going from $3 \AA / \mathrm{s}$ to a $6 \AA / \mathrm{s}$ approach rate. How about the time to expel the layer after a "hole" has nucleated? In a recent measurement by Becker et al., ${ }^{14}$ an OMCTS layer of $25 \mu \mathrm{m}$ radius was expelled in about $2 \mathrm{~s}$. According to Persson et al., ${ }^{20}$ the squeeze-out time is proportional to the area of film that needs to be expelled. Thus, with a $100 \mathrm{~nm}$ radius tip, a layer should be expelled in about $10-100$ $\mu \mathrm{s}$. This is much too fast to be observed in our measurements.

The mechanical behavior of this simple system changes profoundly from liquid to solid, depending on an experimentally imposed (macroscopically long) time scale. The system exhibits a sharp, kinetically induced transition in response to a rather small change in this time scale. This change (a factor of 2) is very small indeed, if we consider thermodynamic arguments where rates are typically exponentially dependent on activation energies (such as the nucleation model discussed above). This suggests that, under confinement, that is, effectively in two dimensions, it is difficult for the molecules to move out of the way of the approaching surfaces except by a slow cooperative process involving a characteristic number of molecules (which may depend on the specific interactions between the molecules). If the molecules are not given enough time, they become jammed, and the system is forced into a nonequilibrium "solid" state, exhibiting high friction (Xhu and Granick) and an elastic response to normal pressure (present work).

How can we estimate the number of molecules that have to act cooperatively in order to arrive at a characteristic time of a few seconds for one complete squeeze-out? A typical molecular relaxation time (i.e., the average time a molecules moves freely before colliding with another molecule) is on the order of $\tau_{0}=$ $10^{-14} \mathrm{~s}$. To move out a layer beneath the tip, the molecules have to move away from a central point, opening up a hole in the layer. Treating the problem as a two-dimensional problem, we can see that the probability that a molecule will move away from a central point, rather than toward it, is approximately $1 / 2$ (since $1 / 2$ of all possible angles of motion will point away from a central point). Thus, the probability that $N$ molecules will all move away from a central point at the same time is on the order of $p_{\mathrm{N}}=(1 / 2)^{N}$. Then the mean time for the event of $N$ molecules moving away from a central point (opening up a hole in the layer) to happen randomly is on the order of $\tau_{0} / p_{\mathrm{N}}$. For this time to be $1.5 \mathrm{~s}, N$ would have to be about 47 molecules. Thus, even a rather small number of cooperatively moving molecules can lead to macroscopic relaxation times.

There are interesting parallels between the research presented here and other systems in which surprisingly long relaxation times have been observed. These include folding/unfolding in biological macromolecules, colloidal suspensions, and glassy systems. In all these cases, slow dynamics arises from the motion of relatively few particles, which are forced to act cooperatively due to internal or external geometric constraints. Close to thermodynamic equilibrium, fluctuations drive the system through various configurations on a complicated free energy landscape, ${ }^{21}$ until the lowest energy state is found and the system can relax.

(20) Persson, B. N. J.; Tosatti, E. Phys. Rev. B 1994, 50, 5590.

(21) Bryngelson, J. D.; Wolynes, P. G. J. Phys. Chem. 1989, 93, 6902. 
Because of this "diffusion" through configuration space, the corresponding relaxation time can be quite long.

However, if the rate of change of some external field is too fast, the system cannot relax, and we observe a "jamming transition", such as that seen in suspensions of colloidal particles, granular materials, ${ }^{22}$ or glassy systems near the glass transition. ${ }^{23}$ The slow dynamics often observed in protein folding has also been described using the idea of a glassy system. ${ }^{21}$ The striking difference is that OMCTS is a simple homogeneous liquid of small globular molecules, while most other systems exhibiting slow dynamics tend to be significantly more complicated.

What are the practical implications of these observations? In macroscopic systems, even in the absence of a lubricant, friction is dominated by confined layers of contaminant hydrocarbon and water layers. ${ }^{24}$ In such systems, lateral motions are typically not well-controlled on a molecular scale, and any shear motion will be accompanied by a normal motion far exceeding $3 \AA / s$. Thus, we could expect that macroscopic friction is partially due to the molecular jamming of lubricant molecules.

In microscopic situations, including nanoelectromechanical

(22) Sanchez-Castillo, F. X.; Anwar, J.; Heyes, D. M. Chem. Mater. 2003, 15, 3417.

(23) Russell, E. V.; Israeloff, N. E. Nature 2000, 408, 695.

(24) He, G.; Müser, M. H.; Robbins, M. O. Science 1999, 284, 1650. systems (NEMS), our findings may provide insight for the management of frictional dissipation. As long as approach rates can be kept very low, lateral friction can be kept very low as well. This should be possible in systems that are approaching molecular dimensions. Furthermore, the fact that under faster approach rates the system behaves elastically may lead to designs that exploit the confined lubricant as a "smart liquid" to control approach rates in small devices.

In summary, we have reported on a simple system that can be forced, under nanoscale geometric confinement, to show collaborative dynamics and surprisingly slow relaxation. If enough time is allowed for relaxation to occur, the system will remain liquidlike. However, if the system is forced at a rate faster than its intrinsic relaxation rate, it will "jam up" and exhibit elastic, solidlike behavior.

Acknowledgment. P.M.H. acknowledges generous support by NSF (MRI Grant DBI-0321011, ANESA Grant INT-0217789, and CAREER Grant DMR-0238943). A.O. and P.M.H. also acknowledge support for international collaborative research through NSF and the Turkish Government (TUBITAK, Grant No. TAG-U/50(102T117)).

LA060504W 